\title{
The Sensitivity of Physiological Measures to Phobic and Non-Phobic Fear Intensity
}

\author{
Tatjana Aue ${ }^{1}$, Marie-Eve Hoeppli ${ }^{2}$, and Camille Piguet ${ }^{1}$ \\ ${ }^{1}$ University of Geneva, Geneva, Switzerland \\ ${ }^{2}$ McGill University, Montreal, Canada
}

\begin{abstract}
Address correspondence to:
Tatjana Aue

Swiss Center for Affective Sciences

7, Rue des Battoirs

1205 Geneva

Switzerland

tatjana.aue@unige.ch
\end{abstract}

Phone: +41 223799802

Fax: +41223799844 
Sensitivity to fear intensity 2

\begin{abstract}
We investigated whether amygdala activation, autonomic responses, respiratory responses, and facial muscle activity (measured over the brow and cheek [fear grin] regions) are all sensitive to phobic versus non-phobic fear and, more importantly, whether effects in these variables vary as a function of both phobic and non-phobic fear intensity. Spider-phobic and comparably low spider-fearful control participants imagined encountering different animals and rated their subjective fear while their central and peripheral nervous system activity was measured. All measures included in our study were sensitive to variations in subjective fear, but were related to different ranges or positions on the subjective fear level continuum. Left amygdala activation, heart rate, and facial muscle activity over the cheek region captured fear intensity variations even within narrowly described regions on the fear level continuum (here within extremely low levels of fear and within considerable phobic fear). Skin conductance and facial muscle activity over the brow region did not capture fear intensity variations within low levels of fear: Skin conductance mirrored only extreme levels of fear, and activity over the brow region distinguished phobic from non-phobic fear but also low-to-moderate and high phobic fear. Finally, differences in respiratory measures distinguished phobic from non-phobic fear with no further differentiation within phobic and non-phobic fear. We conclude that a careful consideration of the measures to be used in an investigation and the population to be examined can be critical in order to obtain significant results.
\end{abstract}

Keywords: phobia, fear intensity, amygdala, autonomic nervous system activity, somatic nervous system activity 
Sensitivity to fear intensity 3

\section{The Sensitivity of Physiological Measures to Phobic and Non-Phobic Fear Intensity}

How sensitive are different physiological measures to variations in subjective fear? Are measures that distinguish different levels of phobic fear also suited to distinguish different levels of non-phobic fear? Do some measures capture variations in subjective fear better than others do? The current article addresses these questions with respect to central nervous system (amygdala) and peripheral nervous system (heart rate, skin conductance, respiration, and facial muscle activity over the brow and cheek regions) activity in spider-phobic and control participants.

Fear responses are mediated by a network of subcortical structures, with the amygdala as the key structure (e.g., Davis \& Lang, 2003; Lang, Bradley, \& Cuthbert, 1998; LeDoux \& Phelps, 2000). The amygdala projects to multiple brain regions, including sensory processing areas, the nucleus basalis, and the hippocampus. Hence, the amygdala can exert great influence on processes such as perception, attention, and memory. Numerous studies revealed evidence for the implication of this brain structure in the initiation of fear responses and fear learning (e.g., Armony \& Dolan, 2002; Hamm \& Weike, 2005; Weike et al., 2005). The amygdala is consistently implicated when individuals experience fear or are confronted with evolutionary fear-relevant stimuli, such as spiders and snakes - irrespective of whether the presented threat is attended to or not (e.g., Morris, Öhman, \& Dolan, 1998, 1999).

In a positron emission tomography study, Carlsson et al. (2004) investigated the neural correlates of viewing masked and unmasked phobic (snakes for snake-fearful and spiders for spider-fearful individuals), evolutionary fear-relevant but nonfeared (snakes for spider-fearful and spiders for snake-fearful individuals), and neutral (mushrooms) pictures. When seen outside awareness, phobic and fear-relevant stimuli were associated with equally strong left amygdala activation, which was interpreted as support for biological preparedness and the existence of a direct, low road for fear processing (LeDoux, 1996). Brain activity for the longer, unmasked 
presentation times, in contrast, clearly distinguished phobic from fear-relevant stimulus material. Whereas the viewing of phobia-related pictures was accompanied by bilateral amygdala activation, this was not the case for the fear-relevant pictures. At the same time, lowered activity was observed in the dorsolateral prefrontal cortex and the right lateral orbitofrontal cortex. This pattern of results was interpreted as increased activation of the fear circuit and reduced top-down control for phobia-related stimulus material when reaching awareness.

Thus, in the aware condition, Carlsson et al. (2004) showed differential central nervous system responding with respect to phobic versus non-phobic fear. ${ }^{1}$ However, to our knowledge, no single study to date has simultaneously investigated the sensitivity of amygdala activation with respect to variations in both non-phobic and phobic fear. The current article aims to fill this gap.

On the peripheral level, fear and phobia have been linked to enhanced physiological responses during the confrontation with a feared object or situation, in particular to autonomic arousal. For this reason, classification systems such as the Diagnostic and Statistical Manual of Mental Disorders (4th ed., test rev.; DSM-IV-TR; American Psychiatric Association, 2000) and the International Statistical Classification of Diseases and Related Health Problems (10th rev.; ICD-10; World Health Organization, 1992) include various physiological symptoms as criteria for phobias or panic attacks (see Roth, 2005, for details). Some of these criteria relate to sympathetic tone (sweating; trembling or shaking; palpitations, pounding heart, or accelerated heart rate), others to respiratory changes associated with hyperventilation (sensations of shortness of breath or smothering; feeling of choking; chest pain or discomfort; feeling dizzy, unsteady, lightheaded, or faint; paresthesias).

Consistent with this picture, high animal fear and animal phobia in humans have been related to increased heart rate and blood pressure, as well as to elevated electrodermal activity, 
during confrontation with the feared animals across diverse laboratory studies (Fredrikson, Sundin, \& Frankenhaeuser, 1985; Globisch, Hamm, Esteves, \& Öhman, 1999; Hamm, Cuthbert, Globisch, \& Vaitl, 1997; Mühlberger, Wiedemann, Herrmann, \& Pauli, 2006; Sarlo, Palomba, Angrilli, \& Stegagno, 2002; Wendt, Lotze, Weike, Hosten, \& Hamm, 2008).

Few studies have investigated facial muscle activity over the brow region associated with frowning (mostly related to the M. Corrugator supercilii) in the context of animal fear or phobia. Knopf and Pössel (2009) found that spider phobics displayed higher increases in activity at this site than did normal controls when they viewed pictures of spiders rather than neutral material (see also Dimberg, Hansson, \& Thunberg, 1998; Wendt et al., 2008).

To our knowledge, only a single study (Dimberg et al., 1998) has investigated the influence of animal fear on facial muscle activity over the cheek region. This is bewildering because this region has been related to the activation of a fear grin across different species (e.g., van Hooff, 1972). Indeed, we (Aue, Flykt, \& Scherer, 2007) observed such fear-grin activation in normal controls during the confrontation with pictures of snakes and spiders. Surprisingly though, Dimberg et al. (1998) reported a decrease, rather than an increase, in activity over the cheek region when high snake-fearful individuals looked at pictures of snakes. Thus, further research on activity over the cheek region with respect to this issue is needed.

Concerning respiration, results are also less clear-cut than for heart rate and skin conductance. Whereas Sarlo et al. (2002) did not find a change in respiration rate when spider phobics were confronted with phobic film stimuli, Gemignani et al. (2000) reported an increased respiration rate in simple phobia (including animal phobia) during hypnotic imagination of phobic images. Findings with respect to other forms of phobia are also divergent concerning respiration rate (e.g., Gerlach et al., 2006; Wilhelm \& Roth, 1998). This inconsistency extends to other measures of respiration (e.g., inspiratory pauses), although the general consensus is that 
there exist at least some deviations in respiration patterns in some phobias, such as driving and flight phobia (e.g., Alpers, Wilhelm, \& Roth, 2005; Wilhelm \& Roth, 1998).

One reason for unreliable observations may be that phobia is not characterized by a fixed set of responses that is automatically triggered when a phobic stimulus is encountered. In fact, phobia may not be reflected similarly across different individuals—or across different variables. Knopf and Pössel (2009) registered physiological responses while spider phobics and normal controls viewed pictures of spiders. For each physiological measure, the authors classified the participants within each group as high, medium, and low responders. Interestingly, only skin conductance and activity over the brow region reliably distinguished between phobics and controls; in both variables, even the low responding phobic individuals displayed higher response magnitudes than did the high responding control individuals. In contrast, with respect to heart rate and cortisol responses, one third to two thirds of the phobic individuals were indistinguishable from the control individuals.

However, why is it that some individuals display enhanced responding in a given variable, whereas others do not? We postulate that at least part of the inconsistencies in results across different studies can be explained by variations in fear levels (i.e., fear intensities)—both across and within individuals. Situations defined as phobia relevant by an experimenter need not be interpreted and experienced in the same manner by the study participants. Only when these individuals experience danger (and subsequently fear) to a certain extent can we expect enhanced physiological changes to occur.

Specifically, we hypothesize that a certain fear intensity onset threshold must be exceeded for changes in physiological measures to be observable. There may also exist a ceiling threshold at which increasing subjective fear levels are no longer associated with increasing physiological response. Moreover, these intensity thresholds may well vary across different physiological 
measures, thus linking the sensitivity of different measures to different ranges and positions on the subjective fear intensity continuum. As a result, a study using only measure A (e.g., heart rate) may suggest differences between two experimental conditions or two groups of participants, whereas another study using only measure B (e.g., respiration) may not.

The aim of the current study was therefore to investigate the sensitivity of diverse physiological measures to variations within phobic and non-phobic fear. We examined two groups of participants: spider phobics, characterized by spider phobia and comparably low levels of snake fear, and a control group, characterized by comparably low levels of both spider and snake fear. Thus, we were able to test whether different physiological measures can (a) distinguish phobic from non-phobic fear (e.g., comparison of responses to spiders in spiderphobic individuals with responses to spiders in control individuals and/or responses to snakes in spider-phobic individuals); (b) distinguish between different levels of phobic fear (e.g., comparison of situations in which spider-phobic individuals are confronted with spiders and experience extreme fear with situations in which spider-phobic individuals are confronted with spiders and experience low-to-moderate fear); and (c) distinguish between different levels of nonphobic fear (e.g., comparison of situations in which spider-phobic and control individuals are confronted with snakes and experience comparably high [non-phobic] fear with situations in which spider-phobic and control individuals are confronted with snakes and experience comparably low [non-phobic] fear). ${ }^{2}$

Participants in our study looked at pictures of spiders, snakes, and birds (unthreatening reference category) and subsequently rated their fear of these animals. In line with earlier research conducted in the area (e.g., Carlsson et al., 2004; Wendt et al., 2008), we hypothesized that spider-phobic participants would display enhanced amygdala activation, autonomic arousal 
(reflected in elevated heart rate and skin conductance), and increased muscle activity over the brow region toward spiders as compared with snakes (and birds).

More important, given its central function in the fear circuit, the amygdala was expected to capture variations within both phobic and non-phobic fear. Heart rate and skin conductance variables were supposed to be characterized by a high sensitivity for variations within phobic fear because they are directly related to a shift from sustained attention to defensive response mobilization and action preparation (especially heart rate, cf. the defense cascade model; Bradley \& Lang, 2007). Sustained attention might prevail in low fear phobic situations, whereas defensive response mobilization and action preparation might be more imminent in high fear phobic situations.

Activity over the brow region was also expected to be sensitive to variations within high levels of subjective fear (i.e., phobic fear) because situations provoking strong fear should be experienced as greatly obstructing the basic human need for safety (see Aue \& Scherer, 2008, 2011, for a link of this measure to goal obstruction). Finally, activity over the cheek region and respiration data were mainly investigated for explorative purposes because of missing or contradicting evidence in the literature, with less strong hypotheses than for the other measures. However, on the basis of $D S M-I V-T R$ criteria, we also predicted hyperventilation and greater tidal volume — signaling greater irregularities in respiration — for spiders than for snakes (and birds) in phobic participants. Activity over the cheek region was predicted to increase for spiders as compared with snakes (and birds) in the spider-phobic group (fear grin; Aue et al., 2007). We had no specific a priori hypotheses regarding the sensitivity of these latter variables for variations within phobic and non-phobic fear.

\section{Method}


Sensitivity to fear intensity 9

\section{Participants}

Participants were recruited via ads placed in university buildings, as well as on university and local websites, and interviewed by telephone to check for $D S M-I V$-TR and $I C D-10$ criteria for the presence or absence of spider phobia (adapted from Mühlberger et al., 2006) and comparably low snake fear. Thirty-six individuals (all female, 18 spider phobics), aged between 19 and 44 years $(M=25.8, S D=5.79)$ took part in the study. One participant in the control group was excluded because she did not follow task instructions, another in the phobic group because of data storage failure.

Fear of spiders and snakes was also assessed by asking the participants to rate their respective fears on a scale from 0 (no fear at all) to 100 (maximal or extreme fear). Spiderphobic participants rated their fear of spiders higher than did control participants, $t(32)=13.80, p$ $<.000001(M \mathrm{~s}=83.2$ and $16.4 ; S D \mathrm{~s}=10.14$ and 15.68 , respectively $)$. The two groups did not differ with respect to their ratings for fear of snakes, $t(32)=-0.27, n s(M \mathrm{~s}=11.5$ and $12.4 ; S D \mathrm{~s}=$ 9.31 and 8.99). Fear of spiders and snakes was further assessed after the experiment by the use of the French translation of the Fear of Spiders Questionnaire (Dan-Glauser, 2008), $t(32)=8.99, p<$ $.000001(M \mathrm{~s}=86.5$ and $23.5 ; S D \mathrm{~s}=28.38$ and 5.58) and the French translation of the Snake Questionnaire (Dan-Glauser, 2008), $t(32)=0.83, n s(M \mathrm{~s}=4.1$ and 3.2; $S D \mathrm{~s}=3.19$ and 2.98). Participants in the two groups did not differ with respect to age, $t(32)=-0.03, n s(M \mathrm{~s}=25.9$ and 25.9; $S D \mathrm{~s}=5.44$ and 6.42).

\section{Stimuli}

Stimuli consisted of 30 pictures displaying spiders and 30 pictures displaying snakes, all taken from a recently created picture base (Dan-Glauser \& Scherer, 2011). Spider and snake pictures were matched for valence, $t(58)=0.08, n s(M s=3.1$ and $3.1 ; S D s=0.94$ and 0.95 , for spiders and snakes, respectively; scale range: 1 [very unpleasant] to 9 [very pleasant]), and for 
arousal ratings, $t(58)=0.03, n s(M \mathrm{~s}=6.1$ and $6.1 ; S D \mathrm{~s}=0.88$ and 0.75 , for spiders and snakes, respectively; scale range: 1 [not arousing at all] to 9 [very arousing]), as assessed in an earlier study (Dan-Glauser \& Scherer, 2011). Thirty additional pictures displaying birds were collected from the Internet.

\section{Setting and Apparatus}

Magnetic resonance imaging (MRI) data were acquired from a 3T scanner (Trio TIM, Siemens, Germany) with the product 12-channel head coil. Peripheral nervous system activity was acquired continuously with the Biopac MP150 System (Goleta, CA, USA). There were different settings for the electrocardiogram, skin conductance, electromyogram (EMG), and respiration channels (see the Dependent Variables subsection for details). Peripheral signals were transferred from the experimental room to the MP150 Acquisition Unit (16 bit A/D conversion) in the control room and stored on computer hard disk. A digital channel received inputs from the presentation computer and recorded on- and offset of the presented stimuli. Visual stimuli were presented on a back projection screen inside the scanner bore using an LCD projector (CPSX1350, Hitachi, Tokyo, Japan). Responses were recorded with a response button box (HH-1×4CR, Current Designs, Inc., Philadelphia, PA, USA). Experimental control was performed by EPrime 2 Professional (Psychology Software Tools, Sharpsburg, PA, USA).

\section{Procedure}

Upon participants' arrival at the laboratory, the nature of the experiment was explained and written informed consent was obtained (protocol approved by the local ethics committee). After participants had thoroughly read the instructions for the task, they performed 10 practice trials with neutral animals only (e.g., goats and frogs) to become familiar with the task. Participants imagined that they were visiting different locations in a forest, with the possibility of encountering different animals (spiders, snakes, or birds) at these same locations. Specifically, in 
each trial, participants saw a picture of a forest location $(1 \mathrm{~s})$, followed by a picture of an animal (spider, snake, or bird; $4 \mathrm{~s}$ ). They then indicated the fear they experienced at the imagination of the scenario on a 17-point scale (from 0\% [no fear at all] to 100\% [extreme, paralyzing fear]; increasing in steps of $6.25 \%) .{ }^{3}$ Thus, ratings were made on a trial-by-trial basis. Responses were given by pressing two buttons of a button box, which moved a slider across a horizontal scale. The 90 experimental trials were presented in random order in two runs of 23 trials and two runs of 22 trials. The intertrial interval was about $15 \mathrm{~s}$. Central (MRI) and peripheral nervous system activity was measured while participants performed the task. ${ }^{4}$

\section{Dependent Variables}

Behavioral data. The dependent behavioral variable comprised the participants' fear estimates for the three types of animals (spiders, snakes, and birds).

\section{Central nervous system data (functional magnetic resonance imaging; fMRI).}

Structural images were acquired with a $T_{1}$-weighted 3D sequence (MPRAGE, TR/TI/TE = 1900/900/2.27 ms, flip angle $=9$ degrees, PAT factor $=2$, voxel dimensions: $1 \mathrm{~mm}$ isotropic, 256 $\times 256 \times 192$ voxels). Functional images were acquired with a $T_{2}{ }^{*}$-weighted EPI sequence $(\mathrm{TR} / \mathrm{TE}$ $=2000 / 30 \mathrm{~ms}$, flip angle $=80$ degrees, PAT factor $=2,64 \times 64$ pixels, $3.2 \times 3.2 \mathrm{~mm}$, 36 slices, 3.2-mm slice thickness, $20 \%$ slice gap). An automatic shimming procedure was performed to minimize inhomogeneities of the static magnetic field. At the beginning of each session, image acquisition started after the recording of three dummy volumes to avoid $T_{1}$ saturation effects.

MRI data were preprocessed and analyzed using SPM8 (Wellcome Department of Imaging Neuroscience, London, UK; http://www.fil.ion.ucl.ac.uk/spm). Functional images were reoriented to the AC-PC line, spatially realigned to the first volume by rigid body transformation, corrected for time differences in slice acquisition using the middle slice in time as reference, spatially normalized to the standard Montreal Neurological Institute EPI template, resampled to 
an isotropic voxel size of $3 \mathrm{~mm}$, and spatially smoothed with an isotropic 8-mm full width at half-maximum Gaussian kernel (Friston et al., 1995).

Peripheral nervous system data. Physiological signals were recorded continuously with a sampling rate of $10000 \mathrm{~Hz}$. Parameterization was performed with AcqKnowledge 4.1 (Biopac, Goleta, CA, USA).

Heart rate. Heart rate (in beats per minute) was assessed by the use of MR-compatible ConMed Cleartrace (ConMed Corporation, Utica, NY, USA) pre-gelled disposable Ag/AgCl electrodes. Electrodes were fixed at a short distance around the heart (Einthoven II). Amplification was set to 500 and the online high-pass filter was set to $0.5 \mathrm{~Hz}$. To eliminate scanner noise, a comb band stop filter was performed offline at $17.5 \mathrm{~Hz}$ (with all harmonics out to Nyquist).

Skin conductance. Electrodermal activity was measured with a constant voltage of $0.5 \mathrm{~V}$, using MR-compatible ConMed Cleartrace pre-gelled disposable Ag/AgCl electrodes. The transducers were placed at the volar surfaces of the medial phalanges of the index and middle fingers of the left hand. Amplification was set to a sensitivity of $5 \mu \mathrm{S} / \mathrm{V}$ and online filters were set to DC and $10 \mathrm{~Hz}$. Offline, the signal was subjected to a low-pass filter of $1 \mathrm{~Hz}$.

Respiration. Respiration was measured with the TSD201 respiratory effort transducer placed around the participants' abdomen. Amplification was set to 10 and online filters were set to DC and $10 \mathrm{~Hz}$. Offline, the signal was subjected to a low-pass filter of $1 \mathrm{~Hz}$.

Facial muscle activity (activity over the cheek region, measured over the $M$. Zygomaticus major; activity over the brow region, measured over the M. Corrugator supercilii). Skin was first cleansed with Con-Zellin (Hartmann, Neuhausen, Switzerland). Two MR-compatible 4-mm Biopac $\mathrm{Ag} / \mathrm{AgCl}$ surface electrodes per site were placed according to the guidelines of Fridlund and Cacioppo (1986). The conducting medium was Biopac Tensive 
Adhesive Gel (GEL103). Online, the EMG signals were amplified by 500 and high-pass filtered $(1 \mathrm{~Hz})$. To eliminate scanner noise, a comb band stop filter was performed offline at $17.5 \mathrm{~Hz}$ (with all harmonics out to Nyquist). The signal was then subjected to a band-pass filter with a low-frequency cutoff of $20 \mathrm{~Hz}$ and a high-frequency cutoff of $400 \mathrm{~Hz}$.

\section{Data Analysis}

Behavioral data. Participants' fear estimates were subjected to an analysis of variance (ANOVA) with the factors Animal (bird, snake, spider) and Group (spider phobic, control). We added the factor Time (early, late ratings) ${ }^{5}$ to test whether subjective fear ratings had been subject to habituation in our experiment. In case of habituation, an analysis of high versus low fear trials could be confounded with habituation effects that we would need to control for in our physiological measures.

\section{Central and peripheral nervous system data.}

FMRI. Statistical analysis was performed using the general linear model for event-related designs in SPM8. Hemodynamic response functions with 16 regressors have been estimated for the whole time series: one regressor for the forest picture, five different regressors for the animal picture presentation phase (spider-low fear, spider-high fear, snake-low fear, snake-high fear, bird), five regressors for the expectancy rating phase (see footnote 2; same event categories as for animal phase), and another five regressors for the fear rating phase (same event categories). Six motion-correction parameters were also added to the model. A high-pass filter of $128 \mathrm{~s}$ was applied to account for low-frequency noise of the scanner and first-order autoregressive corrections for autocorrelation between scans. Effects at each brain voxel were estimated using a least squares algorithm. Parameter estimates describing the mean activity change within the left and right amygdala as provoked by the animal picture presentation were then extracted by 
applying amygdala masks according to the automated anatomical labeling approach of activations (Tzourio-Mazoyer et al., 2002).

Heart rate. (a) Five heart rate scores were computed, one for each of the $4 \mathrm{~s}$ following stimulus presentation and another for the $1 \mathrm{~s}$ following offset of the stimulus presentation $(0-1 \mathrm{~s}$, 1-2 s, 2-3 s, 3-4 s, and 4-5 s, respectively). Heart rate during the $2 \mathrm{~s}$ before picture onset served as baseline. (b) For comparison purposes with the other peripheral variables, a second time interval covered the whole trial.

Skin conductance. Because skin conductance, in general, changes relatively slowly, the first second after stimulus onset was not considered. In addition, skin conductance is often not reliable for short time segments; therefore, skin conductance change was calculated for the entire trial (including the subsequent ratings, thus spanning 1 to $15 \mathrm{~s}$ after picture onset). Mean skin conductance during the $2 \mathrm{~s}$ preceding picture onset was taken as baseline.

Respiration rate and amplitude. Because respiration is also a long latency signal, mean respiration rate and maximal amplitude (as an estimate of tidal volume) ${ }^{6}$ were also calculated for the entire trial. To determine maximal amplitude, we rectified the signal and determined the highest value in the time interval. We did not subtract a baseline as we did for skin conductance and heart rate because $2 \mathrm{~s}$ preceding picture onset was judged to be insufficient.

Facial muscle activity. A logarithmic transformation was undertaken to normalize the distribution, as the EMG measures were characterized by positive skewness. (a) Similar to heart rate scores, EMG scores consisted of mean muscle activity during each of the five 1-s intervals following stimulus onset (overall picture presentation time $+1 \mathrm{~s}$ following picture offset). (b) Given that muscle activity is a short latency signal and changes rapidly over time, we analyzed the first 1-s interval following picture onset in greater detail. Five micro scores were computedone for each 200-ms period during the first 1-s interval after stimulus onset (0-200 ms, 200-400 
ms, $400-600 \mathrm{~ms}, 600-800 \mathrm{~ms}, 800$ to $1,000 \mathrm{~ms}$ ). (c) For comparison purposes with the other peripheral variables, a third time interval covered the whole trial. The time interval of $1 \mathrm{~s}$ before picture onset to picture onset was used as baseline.

Outliers (deviating more than three standard deviations from the mean value of a given participant during a particular time interval) and artifacts were eliminated (making up approximately $1 \%$ of all data). Baseline scores were then subtracted from the scores during and after stimulus presentation (except for respiration) to obtain difference scores describing physiological changes resulting from the presentation of the different stimuli.

Because of technical problems (i.e., related to scanner noise), one control participant was excluded from analyses of activity over the cheek region; two participants (one phobic and one control) were excluded from skin conductance analyses, and four phobic and three control participants were excluded from heart rate analyses.

One ANOVA per variable was conducted for the 2 (Animal: snake, spider) $\times 2$ (Fear Intensity: high, low $)^{7} \times 2$ (Group: phobic, control) mixed-factorial design. Birds were not considered, because our participants consistently reported not fearing them at all. For variables, which were assumed to capture temporal aspects (heart rate and facial EMG), we added the factor Time (five levels).

We were particularly interested in the following results: (a) significant main effect of Fear Intensity with simultaneous absence of any significant interaction comprising the factor Fear Intensity, because this would indicate a measure's general sensitivity for variations in fear levels - irrespective of whether this concerned phobic or non-phobic fear. Such a main effect was predicted for amygdala activation; (b) significant interactions of the factors Animal and Group because this could indicate a measure's capacity to distinguish between phobic and non-phobic fear (e.g., enhanced responding to spiders in spider phobics). In case of the existence of such a 
significant interaction, separate ANOVAs were conducted for phobics and controls to investigate the specific character of the interaction; according to our hypotheses, differences between spiders and snakes would be expected in spider phobics but not in controls. The capacity to distinguish between phobic and non-phobic fear was predicted for all investigated physiological measures; (c) significant interactions comprising the factors Animal, Fear Intensity, and Group, because this could indicate that the differentiation of fear levels in a specific variable might be restricted to phobic or non-phobic fear. Again, in case of the existence of such a significant interaction, separate ANOVAs were conducted for phobics and controls to investigate the type of interaction. Such an interaction was expected for heart rate, skin conductance, and EMG over the brow region, measures that were supposed to well capture variations within phobic but not necessarily within non-phobic fear.

\section{Results}

\section{Behavioral Data}

The ANOVA conducted on the participants' fear ratings revealed a significant main effect of Group, $F(1,32)=19.92, p<.0001$, a significant main effect of Animal, $F(2,64)=145.82, p<$ .000001 , but, most important, a significant interaction of Animal and Group, $F(2,64)=43.88, p$ $<.000001$ (Figure 1). Post hoc Tukey tests revealed that both spider phobics and control participants displayed more fear for snakes and spiders than for birds. The groups did not differ in fear of snakes or fear of birds. Finally, phobic fear in spider phobics was characterized by particularly high fear ratings and could be distinguished from all other experimental conditions.

Regarding the analysis of early versus late trials, contrary to the idea of a habituation of fear responses toward the end of the experiment, we observed higher fear ratings in the late rather than the early trials; main effect of Time, $F(1,32)=5.91, p<.05(M \mathrm{~s}=0.31$ and 0.32 , respectively). This effect varied in both groups, however, as a function of the animal displayed; 
significant interaction between Time and Animal, $F(2,64)=4.37, p<.05$ (interaction between Time, Animal, and Group, $F(2,64)=0.18, n s)$. The $t$ tests for early versus late trials for each animal separately revealed no difference for birds and spiders, $t \mathrm{~s}(33)=-0.36$ and 0.02 , both $n s$, respectively. In contrast, for an unknown reason, late ratings for snakes were characterized by higher fear intensity than were early ratings, $t(33)=-3.23, p<.005$. Given these results, there was no need to control for habituation processes in subjective ratings in the following analyses.

\section{Central and Peripheral Nervous System Data}

To test whether the physiological responses were modulated by subjective fear, for each participant's fear estimates, a median split was conducted for spiders and another for snakes. The experimental trials thus were characterized by comparably low (below median split) or high (above median split) subjective fear $(M \mathrm{~s}=0.26,0.45,0.77$, and 0.93 , for low snake fear, high snake fear, low spider fear, and high spider fear in the phobic group, respectively; $M \mathrm{~s}=0.22$, $0.39,0.21$, and 0.42 , for low snake fear, high snake fear, low spider fear, and high spider fear in the control group, respectively). ${ }^{8}$ An overview of all effects tested is displayed in Table 1. In the following sections, only the most important significant effects are described in further detail.

FMRI. We predicted amygdala activation to distinguish phobic from non-phobic fear, and also to be sensitive to intensity variations within both types of fear. Data for the left amygdala confirmed our hypotheses. Specifically, we found a significant interaction between Animal and Group. Post hoc Tukey tests for this interaction demonstrated higher left amygdala activation in response to spiders than in response to snakes in the spider-phobic group $(p<.0005$, $p$ s for all other comparisons $>.23$ ), reflecting the amygdala's capacity to differentiate between phobic and non-phobic fear. Left amygdala activation was furthermore sensitive to intensity variations within both phobic and non-phobic fear (Figure 2), as indicated by the significance of the main effect for Fear Intensity and the absence of any significant interaction comprising the 
factor Fear Intensity (Table 1). Higher fear levels were generally associated with higher left amygdala activation than were low fear levels.

The picture was a bit different for the right amygdala. Here, we found only a trend for the above-described interaction Animal $\times$ Group (but the same pattern). The significant main effects for Animal and Fear Intensity were qualified by the significant interaction Animal $\times$ Fear Intensity. Post-hoc Tukey tests for this interaction revealed that activity in the right amygdala was increased in the spider-high fear trials as compared to all other trials $(p s<.005)$, with no distinction between the latter $(p s>.83)$. This rather curious interaction resulted from the fact that, for some reason, controls did not display an increase in the high fear snake trials as compared to the low fear snake trials, and the difference between these two conditions was very weak in phobics, as well (see Figure 2). Therefore, right amygdala activation seems to reliably distinguish fear levels on the upper end of the fear continuum, but not consistently on the lower end.

Heart rate. We predicted heart rate to distinguish between phobic and non-phobic fear and, in addition, to be sensitive to variations within phobic fear. Analysis of heart rate data largely confirmed our predictions. Because the results were highly comparable for the different investigated time intervals, only the 5-s interval is described here. The overall ANOVA revealed a significant interaction of Animal $\times$ Fear Intensity $\times$ Group (Figure 2). Consequently, separate ANOVAs were conducted on phobic and control individuals. In phobics, there was no general (main) effect of Fear Intensity, $F(1,12)=1.88, n s$, but a significant interaction of Animal and Fear Intensity, $F(1,12)=7.45, p<.05$. Post hoc Tukey tests yielded significant differences between all pairwise comparisons $(p s<.005)$, except between high and low fear of snakes $(p>$ .56). Thus, in accordance with our expectations, variations in subjective fear level had an impact on heart rate related to phobic (spiders) but not to non-phobic (snakes) stimuli in this group. 
Heart rate further distinguished between phobic (spiders) and non-phobic (snakes) threat in general.

Participants in the control group demonstrated a significant main effect of Fear Intensity, $F(1,12)=6.16, p<.05$ (high $>$ low), which did not vary as a function of the animal concerned $($ interaction Animal $\times$ Fear Intensity, $F(1,12)=1.05, n s)$. Thus, somewhat unexpectedly, in the control group, in contrast to the phobic group, non-phobic fear intensity was reflected in heart rate. $^{9}$

Skin conductance. We expected that, similar to heart rate, skin conductance would distinguish between phobic and non-phobic fear and would, in addition, capture variations within phobic fear. Skin conductance data were only partially consistent with these hypotheses (marginally significant interaction Animal $\times$ Fear Intensity $\times$ Group). When only phobics’ responses were analyzed, there was a main effect of Fear Intensity, $F(1,15)=13.07, p<.005$ (high > low), which was qualified by the significant interaction of Animal and Fear Intensity, $F(1,15)=10.52, p<.01$. Post hoc Tukey tests for this interaction demonstrated significant results for all comparisons, including the high fear spider condition $(p s<.005)$. The remaining comparisons failed to reach significance (all $p \mathrm{~s}>.63$ ). Thus, only partially consistent with our hypotheses, phobic material led to increased mean skin conductance with respect to non-phobic material only when it was highly feared. In the control group, there was no significant effect $($ main effect of Fear Intensity, $F(1,14)=1.47, n s$; interaction Animal $\times$ Fear Intensity, $F(1,14)=$ $0.86, n s)$

Respiration. Both respiration measures had been predicted to distinguish phobic from non-phobic fear. In line with our predictions, post hoc Tukey tests for the significant interactions Animal $\times$ Group demonstrated a difference of phobic versus non-phobic content in the spiderphobic group (respiration rate: $p<.05, p$ s for all other comparisons $>.22$; estimate of tidal 
volume: $p<.005$, other $p$ s $>.93$ ). Respiration rate and the estimate for tidal volume did not reveal any significant modulation by subjective fear level for phobic and non-phobic material.

EMG activity over the brow region. We predicted activity over the brow region to distinguish between phobic and non-phobic fear and, in addition, to capture variations within phobic fear. Our data fully confirmed these predictions. Because the results were highly comparable for the different investigated time intervals, only the 5-s interval is described here. Because of the marginally significant interaction Animal $\times$ Fear Intensity $\times$ Group, the two groups of participants were analyzed separately. The main effect of Fear Intensity survived in phobics, $F(1,16)=9.87, p<.01$, but not in the control group, $F(1,15)=0.24$, ns. In addition, the interaction Animal $\times$ Fear Intensity achieved significance in phobics, $F(1,16)=8.63, p<.01$, but not in controls, $F(1,15)=0.98, n s$. Post hoc Tukey tests for this interaction in the phobic group yielded (marginally) significant differences for all comparisons $(p s<.08)$, except between high and low fear of snakes $(p>.60)$. Thus, consistent with our expectations, phobic material led to increased activity over the brow region, especially when it was highly feared.

EMG activity over the cheek region. Activity over the cheek region had been expected to distinguish between phobic and non-phobic fear-which was supported by our data — and we had no predictions regarding the sensitivity of this variable to variations within phobic and nonphobic fear. Activity over the cheek region during the first second of picture viewing revealed a significant main effect of Fear Intensity (high > low). Higher EMG activity over the cheek region was related to higher subsequent fear ratings, independent of participant population and independent of animal concerned. In addition, as in the case of the left amygdala, we observed no significant interaction comprising the factor Fear Intensity. Consequently, activity over the cheek region during the first second was sensitive to variations within both phobic and non-phobic fear. There was, furthermore, a significant interaction of Time $\times$ Animal $\times$ Group. Therefore, separate 
ANOVAs were conducted on phobics and controls. In phobics, the interaction Time $\times$ Animal achieved significance, $F(4,64)=3.20, p<.05$. Post hoc Tukey tests for this interaction revealed, in accordance with our hypotheses, higher activity at this site for spiders than for snakes during the $800-1,000 \mathrm{~ms}$ period after stimulus onset $(p \mathrm{~s}<.08$, for all comparisons containing responses to spiders during the $800-1,000 \mathrm{~ms}$ period after stimulus onset, except $p>.90$ regarding responses to spiders during the $600-800 \mathrm{~ms}$ period, which can be explained by the fact that the enhancement of activity over the cheek region was already building up during that period; see Figure 3). The ANOVA conducted on controls alone revealed a trend for Fear Intensity, $F(1,14)$ $=3.52, p<.09$.

Higher activity at this site for spiders in spider phobics was maintained during the 0 - to 5s interval following stimulus onset (see also whole trial interval), as revealed by the significant interaction Animal $\times$ Group. Interestingly, though, the main effect of Fear Intensity was not maintained. In sum, this measure differed between phobic and non-phobic threat (as we had predicted) and was furthermore sensitive to variations in fear levels within phobic and nonphobic threat early after stimulus onset.

\section{Discussion}

\section{Differentiation of Phobic Versus Non-Phobic Fear}

We expected all physiological measures to well distinguish phobic and non-phobic fear. This was indeed the case for all but one measure: skin conductance. Only highly feared phobic situations were associated with higher skin conductance than were non-phobic situations.

Thus, our data replicated earlier results reported by different research groups (amygdala: Carlsson et al., 2004; autonomic arousal: Fredrikson et al., 1985; Globisch et al., 1999; Hamm et al., 1997; Mühlberger et al., 2006; Sarlo et al., 2002; Wendt et al., 2008; activity over the brow region: Knopf \& Pössel, 2009; Wendt et al., 2008). Beyond that, we observed respiratory changes 
characteristic of a response to phobic stimulus material. Our phobic participants displayed increased respiration rates, and at the same time increased tidal volume, when they were confronted with pictures of spiders.

Most important to the distinction between phobic and non-phobic fear, phobic participants in our study displayed increased activity over the cheek region when they were presented with phobia-relevant pictures. This result is in line with earlier results concerning pictures displaying phylogenetic threat such as snakes and spiders (Aue et al., 2007) in a group of participants unscreened for animal fear, as well as with the idea that increased activity at that site constitutes a residuum of a so-called fear grin (Van Hooff, 1972; see also Harrigan \& O’Connell, 1996, for the description of a "nonenjoyment smile" in fear). That Dimberg et al. (1998) observed a decrease rather than an increase in activity over the cheek region when snake-fearful individuals viewed pictures of snakes may lie in the fact that their comparison condition comprised pictures of flowers. Flowers, instead of being neutral (as is often assumed) may be experienced as positive. Presenting a flower may thus provoke a smile; presenting a flower before a snake, then, might result in decreasing activity over this site when viewing the snake.

Of importance, our data do not imply that all of our measures reliably distinguished all of our phobic participants from all of our control participants. Instead of using the individual comparison approach that Knopf and Pössel (2009) used to demonstrate the differential capacity of different measures to distinguish phobics from controls, we used an ANOVA approach and contrasted conditions across and within these groups. This may explain why Knopf and Pössel found skin conductance to reliably distinguish between phobics and controls, but we observed increased skin conductance only in the high fear spider condition in phobics. ${ }^{10}$ The same applies to results for heart rate, for which Knopf and Pössel found only a restricted capacity to distinguish between phobics and controls. ${ }^{11}$ 


\section{Sensitivity of Physiological Measures for Variations in Fear Intensity}

The specific aim of this study was to test whether response magnitudes of the different physiological measures vary as a function of reported fear intensity - both for phobic and nonphobic fear. Alpers et al. (2005) reported high levels of reported anxiety to decrease over time, which was accompanied by decreased autonomic arousal. However, their observation cannot rule out that reported fear level and physiological responses are related for the simple reason that both types of responses are subject to independent habituation processes.

The participants' fear level did not decrease over the time course of our own experiment. Therefore, differences between high and low levels of fear cannot be attributed to greater physiological habituation in the case of low fear. Specifically, we had two variables, left amygdala activation and activity over the cheek region, that differentiated between phobic and non-phobic threat (e.g., spiders vs. snakes in phobics), but also between high and low intensity within both phobic and non-phobic fear (in the phobic as well as in the control group). Heart rate responses were almost similar, with the only exception being that they did not capture variations in fear levels for non-phobic threat in our phobic participants - somewhat unexpectedly, though, they did so in the control participants. Together, these three variables varied as a function of fear intensity at both extremes (low fear and extreme phobic fear).

Activity over the brow region distinguished between phobic and non-phobic threat and also between extreme and comparably low-to-moderate fear of spiders in the phobic group. It did not, however, distinguish different levels of non-phobic fear. Results were highly comparable for skin conductance. In contrast to activity over the brow region, however, only the highly feared phobic material (but not the low-to-moderately feared phobic material) led to measurable increases in skin conductance. Therefore, it seems that both activity over the brow region and skin conductance discriminate well between fear levels on the upper end of the fear intensity 
continuum investigated in our study, with skin conductance being characterized by a higher threshold than activity over the brow region.

Results for heart rate and skin conductance can be interpreted in the context of the defense cascade model of fear (see Bradley \& Lang, 2007, for a description). According to this model, we would have expected heart rate to display no change or to display bradycardia in the case of weakly arousing situations (e.g., low snake fear in phobics), but to suddenly shift to tachycardia in highly arousing situations (e.g., high spider fear in phobics). Our heart rate data in phobics (but not necessarily in controls) are consistent with these predictions. Also, our skin conductance data seem to be consistent with the model, with the highest emotional arousal (high spider fear in phobics) being associated with the highest skin conductance. Thus, our phobic participants may have been characterized by sustained attention in the low fear situations but by defensive response preparation, particularly in the high phobic fear situations. However, not all participants in the phobic group may have responded in the same way (cf. Knopf \& Pössel, 2009), especially because high and low fear certainly did not mean the same to every participant.

Respiratory measures in this study distinguished phobic and non-phobic stimuli with no further differentiation for fear intensity variations within these two stimulus categories. Finally, the result for the right amygdala was somewhat puzzling. It showed a trend for distinguishing between phobic and non-phobic fear, but also an interaction of Animal and Fear Intensity independent of participant population. We have no conclusive explanation for the latter observation. The significant interaction arose because both groups of participants showed no differentiation in right amygdala activation between high and low fear of snakes (but they did so in the case of spiders). This might be related to our behavioral data that revealed higher fear levels for snakes during the late rather than during the early experimental trials. Late trials, in turn, are generally characterized by stronger physiological habituation than early trials. 
Habituation processes may have been strongest in the right amygdala (compared with the other physiological measures) and thus may have hidden differences between high and low fear of snakes.

To summarize, whereas left amygdala activation, heart rate, and muscle activity over the cheek site appear to capture fear intensity variations even within narrowly described regions on the fear level continuum (here within extremely low levels of fear and within considerable fear), skin conductance and EMG activity over the brow region do not seem to capture fear intensity variations within low levels of fear, with skin conductance mirroring only extreme levels of fear. Finally, differences in respiratory measures distinguished phobic from non-phobic fear with no differentiation within phobic and non-phobic fear and therefore are characterized by a lower onset threshold than skin conductance. The status of the right amygdala remains unclear. Differences between left and right amygdala functioning have been reported before. Carlsson et al. (2004) found the left— but not the right—amygdala to be sensitive to masked phobic and nonphobic fear-relevant stimuli (see also Morris, Öhman, \& Dolan, 1998, 1999, for the influence of experimental modulations of visual attention on right vs. left amygdala activation and connectivity). These observations suggest that the left amygdala may indeed be characterized by overall greater sensitivity to variations in fear intensity, whereas the right amygdala's sensitivity is limited to specific attentional requirements. Because all of our participants knew that the study was on spider phobia, even controls may have attended differently to spiders than to snakes.

\section{Conclusion}

In the current study, we demonstrated the utility of a variety of physiological variables to distinguish phobia-relevant from non-phobia-relevant stimulation. More important, these variables differed in their sensitivity at different levels of the fear intensity continuum that we investigated. Thus, depending on the fear levels of different groups included in a study, a given 
Sensitivity to fear intensity 26

variable can display differences between animals and groups or not. This observation may account for a considerable number of inconsistencies found in the literature. Depending on the distribution of participants' fear levels in a study, we would expect particular variables to display significant results and others not. Therefore, a careful consideration of the measures to be used in an investigation and the population to be examined can be critical in order to obtain significant results. 


\section{References}

Alpers, G. W., Wilhelm, F. H., \& Roth, W. T. (2005). Psychophysiological assessment during exposure in driving phobic patients. Journal of Abnormal Psychology, 114, 126-139.

American Psychiatric Association. (2000). Diagnostic and statistical manual of mental

disorders (4th ed., text rev.). Washington, DC: Author.

Armony, J. L., \& Dolan, R. J. (2002). Modulation of spatial attention by fear-conditioned stimuli: An event-related fMRI study. Neuropsychologia, 40, 817-826.

Aue, T., Flykt, A., \& Scherer, K. R. (2007). First evidence for differential and sequential efferent effects of goal relevance and goal conduciveness appraisal. Biological Psychology, 74, 347-357.

Aue, T., \& Hoeppli, M. E. (2012). Evidence for an encounter expectancy bias in fear of spiders. Cognition and Emotion, 26, 727-736.

Aue, T., \& Scherer, K. R. (2008). Appraisal-driven somatovisceral response patterning: Effects of intrinsic pleasantness and goal conduciveness. Biological Psychology, 79, 158-164.

Aue, T., \& Scherer, K. R. (2011). Effects of intrinsic pleasantness and goal conduciveness appraisals on somatovisceral responding: Somewhat similar, but not identical. Biological Psychology, 86, 65-73.

Bradley, M. M., \& Lang, P. (2007). Emotion and motivation. In J. T. Cacioppo, L. G. Tassinary, and G. G. Berntson (Eds.), Handbook of psychophysiology (3 ${ }^{\text {rd }}$ ed., pp 581-607). New York, NY: Cambridge University Press.

Carlsson, K., Petersson, K. M., Lundqvist, D., Karlsson, Ingvar, M., \& Öhman, A. (2004). Fear and the amygdala: Manipulation of awareness generates differential cerebral responses to phobic and fear-relevant (but nonfeared) stimuli. Emotion, 4, 340-353.

Dan-Glauser, E. S. (2008). Vivre son émotion: Mécanismes d'induction et particularités 
Sensitivity to fear intensity 28

physiologiques d'un processus émotionnel débouchant sur un sentiment subjectif.

(Unpublished doctoral dissertation). University of Geneva, Geneva, Switzerland.

Dan-Glauser, E. S., \& Scherer, K. R. (2011). The Geneva Affective PicturE Database (GAPED):

A new 730 picture database focusing on valence and normative significance. Behavior Research Methods, 43, 468-477.

Davis, M., \& Lang, P. J. (2003). Emotion. In M. Gallagher \& R. J. Nelson (Eds.), Handbook of psychology: Vol. 3. Biological psychology (pp. 405 - 439). New York, NY: Wiley.

Dimberg, U., Hansson, G., \& Thunberg, M. (1998). Fear of snakes and facial reactions: A case of rapid responding. Scandinavian Journal of Psychology, 39, 75-80.

Flykt, A. (2005). Visual search with biological threat stimuli: Accuracy, reaction times, and heart rate changes. Emotion, 5, 349-353.

Fredrikson, M., Sundin, Ö., \& Frankenhaeuser, M. (1985). Cortisol excretation during the defense reaction in humans. Psychosomatic Medicine, 47, 313-319.

Fridlund, A. J., \& Cacioppo, J. T. (1986). Guidelines for human electromyographic research. Psychophysiology, 23, 567-589.

Friston, K. J., Holmes, A. P., Worsley, K. J., Poline, J. B., Frith, C. D., \& Frackowiak, R. S. J. (1995). Statistical parametric maps in functional imaging: A general linear approach. Human Brain Mapping, 2, 189-210.

Gemignani, A., Santarcangelo, E., Sebastiani, L., Marchese, C., Mammoliti, R., Simoni, A., \& Ghelarducci, B. (2000). Changes in autonomic and EEG patterns induced by hypnotic imagination of aversive stimuli in man. Brain Research Bulletin, 53, 105-111.

Gerlach, A. L., Spellmeyer, G., Vögele, C., Huster, R., Stevens, S., Hetzel, G., \& Deckert, J. (2006). Blood-injury phobia with and without a history of fainting: Disgust sensitivity does not explain the fainting response. Psychosomatic Medicine, 68, 331-339. 
Globisch, J., Hamm, A. O., Esteves, F., \& Öhman, A. (1999). Fear appears fast: Temporal course of startle reflex potentiation in animal fearful subjects. Psychophysiology, 36, 66-75.

Hamm, A. O., Cuthbert, B. N., Globisch, J., \& Vaitl, D. (1997). Fear and the startle reflex: Blink modulation and autonomic response patterns in animal and mutilation fearful subjects. Psychophysiology, 34, 97-107.

Hamm, A., \& Weike, A. I. (2005). The neuropsychology of fear learning and fear regulation. International Journal of Psychophysiology, 57, 5-14.

Harrigan, J. A., \& O’Connell, D. M. (1996). How do you look when feeling anxious? Facial displays of anxiety. Personality and Individual Differences, 21, 205-212.

Knopf, K., \& Pössel, P. (2009). Individual-response differences in spider phobia: Comparing phobic and non-phobic women of different reactivity levels. Anxiety, Stress, and Coping, $22,39-55$.

Lang, P. J., Bradley, M. M., \& Cuthbert, B. N. (1998). Emotion, motivation and anxiety: Brain mechanisms and psychophysiology. Biological Psychiatry, 44, 1248-1263.

LeDoux, J. E. (1996). The emotional brain. New York, NY: Simon \& Schuster.

LeDoux, J. E., \& Phelps, E. A. (2000). Emotional networks in the brain. In M. Lewis \& J. M. Haviland-Jones (Eds.), Handbook of emotions (pp. 157-172). New York, NY: Guilford Press.

Morris, J. S., Öhman, A., \& Dolan, R. J. (1998). Conscious and unconscious emotional learning in the human amygdala, Nature, 393, 467-470.

Morris, J. S., Öhman, A., \& Dolan, R. J. (1999). A subcortical pathway to the right amygdala mediating "unseen" fear. Proceedings of the National Academy of Sciences of the United States of America, 96, 1680-1685. 
Sensitivity to fear intensity 30

Mühlberger, A., Wiedemann, G., Herrmann, M. J., \& Pauli, P. (2006). Phylo- and ontogenetic fears and the expectation of danger: Differences between spider- and flight-phobic subjects in cognitive and physiological responses to disorder-specific stimuli. Journal of Abnormal Psychology, 115, 580-589.

Roth, W. T. (2005). Physiological markers for anxiety: Panic disorder and phobias. International Journal of Psychophysiology, 58, 190-198.

Sarlo, M., Palomba, D., Angrilli, A., \& Stegagno, L. (2002). Blood phobia and spider phobia: Two specific phobias with different autonomic cardiac modulations. Biological Psychology, 60, 91-108.

Tzourio-Mazoyer, N., Landeau, B., Papathanassiou, D., Crivello, F., Etard, O., Delcroix, N., .. . Joliot, M. (2002). Automated anatomical labeling of activations in SPM using a macroscopic anatomical parcellation of the MNI MRI single-subject brain. Neuroimage, 15, 273-289.

Van Hooff, J. A. (1972). A comparative approach to the phylogeny of laughter and smiling. In R. Hinde (Ed.), Non-verbal communication (pp. 209-241). Cambridge, England: Cambridge University Press.

Weike, A. I., Hamm, A., Schupp, H. T., Runge, U., Schröder, H. W. S., \& Kessler, C. (2005). Fear conditioning following unilateral temporal lobectomy: Dissociation of conditioned startle potentiation and autonomic learning. The Journal of Neuroscience, 25, 1111711124.

Wendt, J., Lotze, M., Weike, A. I., Hosten, N., \& Hamm, A. O. (2008). Brain activation and defensive response mobilization during sustained exposure to phobia-related and other affective pictures in spider phobia. Psychophysiology, 45, 205-215. 
Wilhelm, F. H., \& Roth, W. T. (1998). Taking the laboratory to the skies: Ambulatory assessment of self-report, autonomic, and respiratory responses in flying phobia. Psychophysiology, 35, 596-606.

World Health Organization. (1992). International classification of diseases and related health problems (10th rev.). Geneva, Switzerland: Author. 
Sensitivity to fear intensity 32

\section{Author Note}

Tatjana Aue, University of Geneva, Geneva, Switzerland; Marie-Eve Hoeppli, McGill

University, Montreal, Canada; Camille Piguet, University of Geneva, Geneva, Switzerland.

This research was supported by grant PZ00P1_121590 of the Swiss National Science

Foundation to Tatjana Aue.

Correspondence concerning this article should be addressed to Tatjana Aue, Swiss Center

for Affective Sciences, University of Geneva, Rue des Battoirs 7, 1205 Geneva, Switzerland. E-

mail: tatjana.aue@unige.ch 


\section{Footnotes}

${ }^{1}$ Stimuli provoking non-phobic fear have been termed fear-relevant but nonfeared by the authors. However, to a certain extent, calling something fear-relevant but nonfeared seems contradictory. Given that these stimuli nevertheless activated the fear circuit in the unmasked condition and given their association with biological preparedness, we prefer to use the term nonphobic fear when referring to this category.

${ }^{2}$ High and low phobic fear refer to different fear intensities than high and low non-phobic fear.

${ }^{3}$ Our participants also rated their expectancy of encountering the animal presented if they were rendering themselves to the forest location in question. Detailed analyses of these expectancy ratings (without any reference to physiological data and irrelevant to the current research questions) can be found elsewhere (Aue \& Hoeppli, 2012).

${ }^{4}$ Given space limits and the simultaneous focus on peripheral physiological responses, discussion of the MRI data is limited to amygdala activations.

${ }^{5}$ Early, here, referred to the first 15 experimental trials of each Animal category, whereas late referred to the last 15 experimental trials of each Animal category.

${ }^{6}$ We included the somewhat "uncommon" respiration amplitude as an estimate of tidal volume to account for the type of intermittent respiration that is often observed in phobia; this type is characterized by high frequency, but can, at the same time, include high depth.

${ }^{7}$ For each participant's fear estimates, a median split was conducted for spiders and another for snakes.

${ }^{8}$ Birds were not considered because our participants said they did not fear them at all. However, we also conducted analyses including birds - comparable to the behavioral data — on our physiological variables. All of the variables that we investigated clearly distinguished phobic 
from other stimuli. Interestingly, virtually no differences were found between phylogenetic threat stimuli not associated with phobic fear (i.e., snakes in the phobic group and both snakes and spiders in the control group) on the one hand and birds on the other hand. There was just a trend for a higher respiration rate for snakes than for birds in spider phobics. The control group did not exhibit any differential activity between phylogenetic threat stimuli and birds.

${ }^{9}$ Recall that the levels for non-phobic fear did not differ between phobics and controls.

${ }^{10}$ In addition, these authors also analyzed skin conductance responses instead of mean skin conductance.

${ }^{11}$ That our participants saw a picture of a forest during the baseline period should be taken into consideration. It could be argued that this is problematic because phobic participants might experience anticipatory anxiety, therefore questioning the validity of the baseline for the subsequent fear response. To investigate this issue, we tested for group differences in physiological responding during the baseline period. However, the only variable that demonstrated baseline group differences was heart rate, with a higher heart rate for phobics than for controls, $t(25)=3.08, p<.01$. Thus, anticipatory anxiety cannot account for the effects we observed here. 
Table 1

Effects in the ANOVAs for the different physiological measures

\section{Left Amygdala $\quad$ Right Amygdala}

\begin{tabular}{|c|c|c|c|c|c|c|}
\hline Effects & $d f$ & $F$ & $p$ & $d f$ & $F$ & $p$ \\
\hline Animal & 1,31 & 13.49 & $<.001$ & 1,31 & 7.87 & $<.01$ \\
\hline Animal $\times$ Group & 1,31 & 8.48 & $<.01$ & 1,31 & 3.32 & $=.08$ \\
\hline Fear & 1,31 & 11.03 & $<.005$ & 1,31 & 10.10 & $<.005$ \\
\hline Fear $\times$ Group & 1,31 & 0.94 & $n s$ & 1,31 & 0.17 & $n s$ \\
\hline \multicolumn{7}{|l|}{ Time $\times$ Animal } \\
\hline \multicolumn{7}{|l|}{ Time $\times$ Animal $\times$ Group } \\
\hline \multicolumn{7}{|l|}{ Time $\times$ Fear } \\
\hline \multicolumn{7}{|l|}{ Time $\times$ Fear $\times$ Group } \\
\hline Animal $\times$ Fear & 1,31 & 2.18 & $n s$ & 1,31 & 6.32 & $<.05$ \\
\hline Animal $\times$ Fear $\times$ Group & 1,31 & 0.84 & $n s$ & 1,31 & 0.68 & $n s$ \\
\hline \multicolumn{7}{|l|}{ Time $\times$ Animal $\times$ Fear } \\
\hline Time $\times$ Animal $\times$ Fear $\times$ Group & & & & & & \\
\hline
\end{tabular}


Table 1 (continued)

\section{Heart Rate}

(5 s)
Heart Rate

(whole trial)
Skin Conductance

(whole trial)

\begin{tabular}{|c|c|c|c|c|c|c|c|c|c|}
\hline Effects & $d f$ & $F$ & $p$ & $d f$ & $F$ & $p$ & $d f$ & $F$ & $p$ \\
\hline Animal & 1,24 & 4.82 & $<.05$ & 1,24 & 2.18 & $n s$ & 1,29 & 5.73 & $<.05$ \\
\hline Animal $\times$ Group & 1,24 & 4.16 & $=.05$ & 1,24 & 6.42 & $<.05$ & 1,29 & 6.92 & $<.05$ \\
\hline Fear & 1,24 & 4.42 & $<.05$ & 1,24 & 3.90 & $=.06$ & 1,29 & 3.76 & $=.06$ \\
\hline Fear $\times$ Group & 1,24 & 0.24 & $n s$ & 1,24 & 0.10 & $n s$ & 1,29 & 12.33 & $<.005$ \\
\hline Time $\times$ Animal & 4,96 & 5.57 & $<.0005$ & & & & & & \\
\hline Time $\times$ Animal $\times$ Group & 4,96 & 7.64 & $<.00005$ & & & & & & \\
\hline Time $\times$ Fear & 4,96 & 0.59 & $n s$ & & & & & & \\
\hline Time $\times$ Fear $\times$ Group & 4,96 & 0.10 & $n s$ & & & & & & \\
\hline Animal $\times$ Fear & 1,24 & 0.07 & $n s$ & 1,24 & 8.38 & $<.01$ & 1,29 & 9.66 & $<.005$ \\
\hline Animal $\times$ Fear $\times$ Group & 1,24 & 4.46 & $<.05$ & 1,24 & 14.64 & $<.001$ & 1,29 & 3.95 & $=.06$ \\
\hline Time $\times$ Animal $\times$ Fear & 4,96 & 0.07 & $n s$ & & & & & & \\
\hline Time $\times$ Animal $\times$ Fear $\times$ Group & 4,96 & 1.33 & $n s$ & & & & & & \\
\hline
\end{tabular}


Table 1 (continued)

\begin{tabular}{|c|c|c|c|c|c|c|}
\hline \multirow[b]{2}{*}{ Effects } & \multicolumn{3}{|c|}{$\begin{array}{l}\text { Respiration Rate } \\
\text { (whole trial) }\end{array}$} & \multicolumn{3}{|c|}{$\begin{array}{l}\text { Tidal volume } \\
\text { (whole trial) }\end{array}$} \\
\hline & $d f$ & $F$ & $p$ & $d f$ & $F$ & $p$ \\
\hline Animal & 1,31 & 6.16 & $<.05$ & 1,31 & 6.91 & $<.05$ \\
\hline Animal $\times$ Group & 1,31 & 4.87 & $<.05$ & 1,31 & 8.75 & $<.01$ \\
\hline Fear & 1,31 & 0.13 & $n s$ & 1,31 & 0.08 & $n s$ \\
\hline Fear $\times$ Group & 1,31 & 0.22 & $n s$ & 1,31 & 0.00 & ns \\
\hline Time $\times$ Animal & & & & & & \\
\hline Time $\times$ Animal $\times$ Group & & & & & & \\
\hline Time $\times$ Fear & & & & & & \\
\hline Time $\times$ Fear $\times$ Group & & & & & & \\
\hline Animal $\times$ Fear & 1,31 & 0.93 & $n s$ & 1,31 & 0.02 & $n s$ \\
\hline Animal $\times$ Fear $\times$ Group & 1,31 & 0.61 & $n s$ & 1,31 & 0.11 & $n s$ \\
\hline Time $\times$ Animal $\times$ Fear & & & & & & \\
\hline Time $\times$ Animal $\times$ Fear $\times$ Group & & & & & & \\
\hline
\end{tabular}


Table 1 (continued)

\section{$\begin{array}{lll}\text { EMG brow } & \text { EMG brow } & \text { EMG brow }\end{array}$ \\ (1 s) (5 s)}

\begin{tabular}{|c|c|c|c|c|c|c|c|c|c|}
\hline Effects & $d f$ & $F$ & $p$ & $d f$ & $F$ & $p$ & $d f$ & $F$ & $p$ \\
\hline Animal & 1,31 & 5.34 & $<.05$ & 1,31 & 12.97 & $<.005$ & 1,31 & 17.42 & $<.0005$ \\
\hline Animal $\times$ Group & 1,31 & 2.75 & $n s$ & 1,31 & 11.23 & $<.005$ & 1,31 & 8.75 & $<.01$ \\
\hline Fear & 1,31 & 2.02 & $n s$ & 1,31 & 8.28 & $<.01$ & 1,31 & 9.36 & $<.005$ \\
\hline Fear $\times$ Group & 1,31 & 4.44 & $<.05$ & 1,31 & 5.61 & $<.05$ & 1,31 & 3.61 & $=.06$ \\
\hline Time $\times$ Animal & 4,124 & 5.62 & $<.0005$ & 4,124 & 4.10 & $<.005$ & & & \\
\hline Time $\times$ Animal $\times$ Group & 4,124 & 8.06 & $<.000001$ & 4,124 & 2.79 & $<.05$ & & & \\
\hline Time $\times$ Fear & 4,124 & 3.82 & $<.01$ & 4,124 & 2.68 & $<.05$ & & & \\
\hline Time $\times$ Fear $\times$ Group & 4,124 & 2.71 & $<.05$ & 4,124 & 4.01 & $<.005$ & & & \\
\hline Animal $\times$ Fear & 1,31 & 2.35 & $n s$ & 1,31 & 8.81 & $<.01$ & 1,31 & 9.12 & $<.01$ \\
\hline Animal $\times$ Fear $\times$ Group & 1,31 & 0.18 & $n s$ & 1,31 & 3.68 & $=.06$ & 1,31 & 4.36 & $<.05$ \\
\hline Time $\times$ Animal $\times$ Fear & 4,124 & 1.69 & $n s$ & 4,124 & 0.56 & $n s$ & & & \\
\hline Time $\times$ Animal $\times$ Fear $\times$ Group & 4,124 & 4.87 & $<.005$ & 4,124 & 1.13 & $n s$ & & & \\
\hline
\end{tabular}


Table 1 (continued)

\begin{tabular}{|c|c|c|c|c|c|c|c|c|c|}
\hline \multirow[b]{2}{*}{ Effects } & \multicolumn{3}{|c|}{$\begin{array}{l}\text { EMG cheek } \\
\text { (1 s) }\end{array}$} & \multicolumn{3}{|c|}{$\begin{array}{c}\text { EMG cheek } \\
(5 \mathrm{~s})\end{array}$} & \multicolumn{3}{|c|}{$\begin{array}{l}\text { EMG cheek } \\
\text { (whole trial) }\end{array}$} \\
\hline & $d f$ & $F$ & $p$ & $d f$ & $F$ & $p$ & $d f$ & $F$ & $p$ \\
\hline Animal & 1,30 & 1.26 & $n s$ & 1,30 & 5.96 & $<.05$ & 1,30 & 6.37 & $<.05$ \\
\hline Animal $\times$ Group & 1,30 & 5.27 & $<.05$ & 1,30 & 6.78 & $<.05$ & 1,30 & 6.06 & $<.05$ \\
\hline Fear & 1,30 & 4.29 & $<.05$ & 1,30 & 0.75 & $n s$ & 1,30 & 0.89 & $n s$ \\
\hline Fear $\times$ Group & 1,30 & 0.60 & $n s$ & 1,30 & 0.25 & $n s$ & 1,30 & 0.21 & $n s$ \\
\hline Time $\times$ Animal & 4,120 & 2.29 & $=.06$ & 4,120 & 2.82 & $<.05$ & & & \\
\hline Time $\times$ Animal $\times$ Group & 4,120 & 2.48 & $<.05$ & 4,120 & 1.92 & $n s$ & & & \\
\hline Time $\times$ Fear & 4,120 & 1.57 & $n s$ & 4,120 & 0.80 & $n s$ & & & \\
\hline Time $\times$ Fear $\times$ Group & 4,120 & 0.46 & $n s$ & 4,120 & 0.63 & $n s$ & & & \\
\hline Animal $\times$ Fear & 1,30 & 0.75 & $n s$ & 1,30 & 0.23 & $n s$ & 1,30 & 0.00 & $n s$ \\
\hline Animal $\times$ Fear $\times$ Group & 1,30 & 0.16 & $n s$ & 1,30 & 0.06 & $n s$ & 1,30 & 1.32 & $n s$ \\
\hline Time $\times$ Animal $\times$ Fear & 4,120 & 0.83 & $n s$ & 4,120 & 0.62 & $n s$ & & & \\
\hline Time $\times$ Animal $\times$ Fear $\times$ Group & 4,120 & 0.39 & $n s$ & 4,120 & 0.14 & $n s$ & & & \\
\hline
\end{tabular}

Note. Main effects of Time and Group as well as the interaction effects Time $\times$ Group are not reported. Boldface numbers refer to significant $(\mathrm{p}<$ .05) effects. EMG = electromyogram. 


\section{Figure Captions}

Figure 1. Fear ratings as a function of Group, Animal, and Time (early vs. late trials). Error bars depict standard errors. Scale ranging from 0 (no fear at all) to 100\% (extreme, paralyzing fear).

Figure 2. Changes in amygdala activation and peripheral nervous system activity as a function of Group, Animal, and Fear Intensity. Error bars depict standard errors. Amygdala: response evoked by picture onset; heart rate and electromyogram (EMG) brow region: average response during 0-5 s following stimulus onset; EMG cheek region: average response during 0-1 s following stimulus onset; skin conductance and respiration measures: average response of whole trial.

Figure 3. Changes in activity over the cheek region in spider-phobic individuals during the first second after stimulus onset as a function of Animal and Time. Error bars depict standard errors. $\mathrm{EMG}=$ electromyogram. 


\section{Fear rating in $\%$}

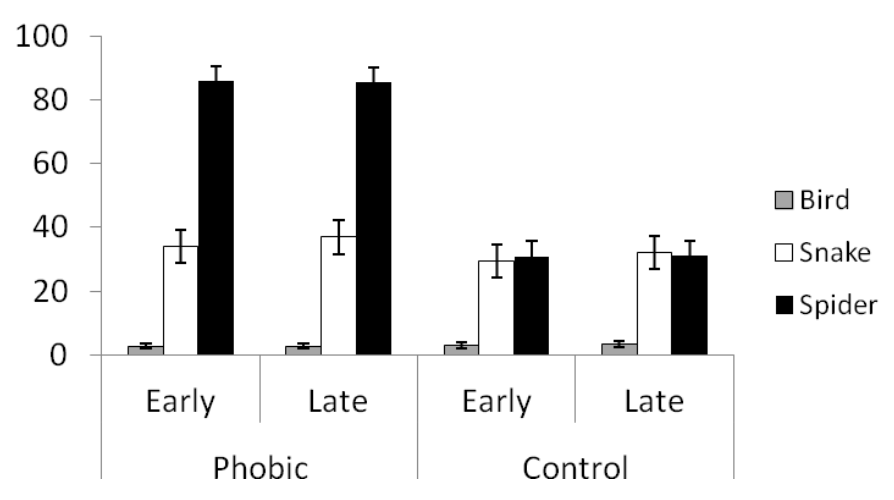


Left amygdala activation

estimated response

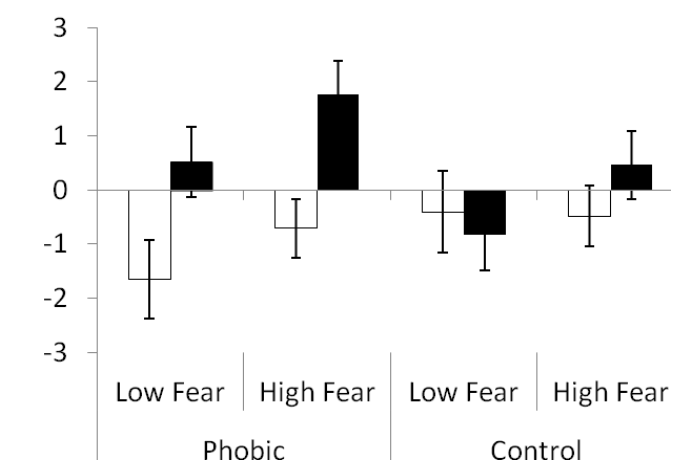

Heart rate

in beats per minute

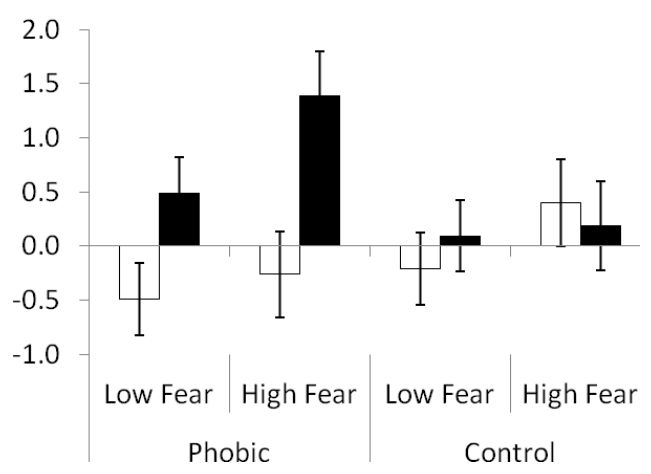

Respiration rate

in cycles per minute

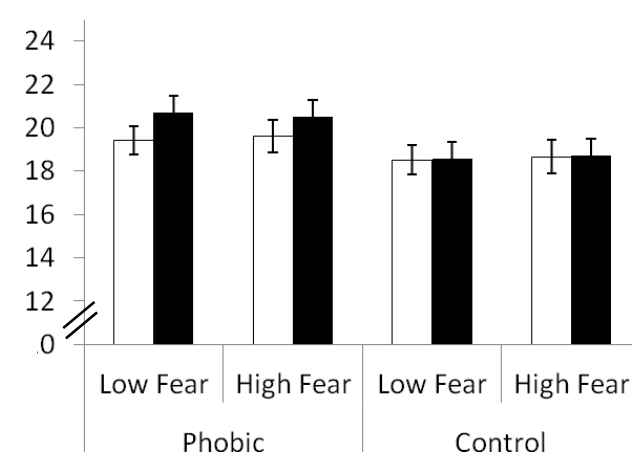

\section{EMG brow region}

in volts (logarithmic transformation)

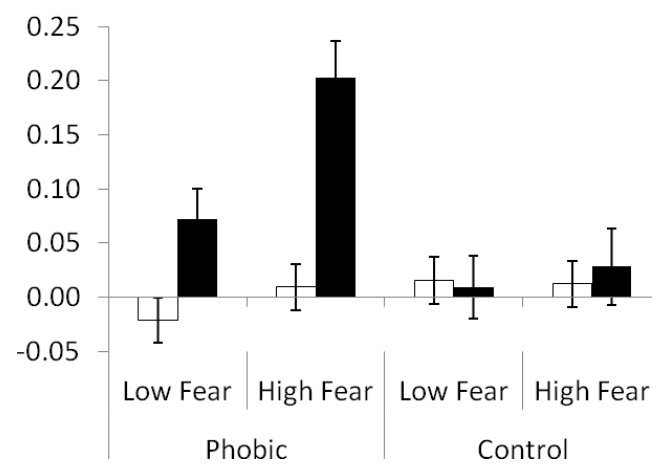

Right amygdala activation

estimated response

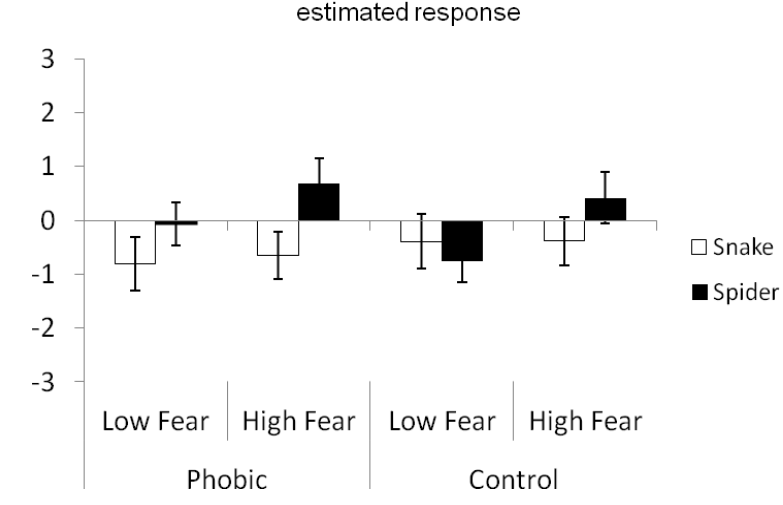

Skin conductance

in microsiemens

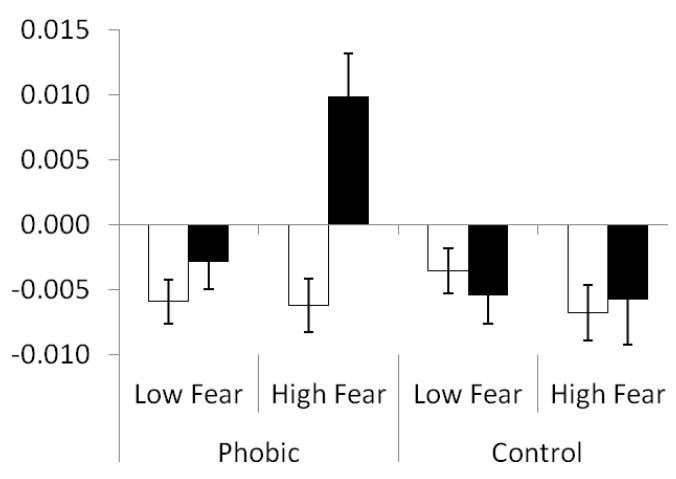

Respiration est. tidal volume in volts

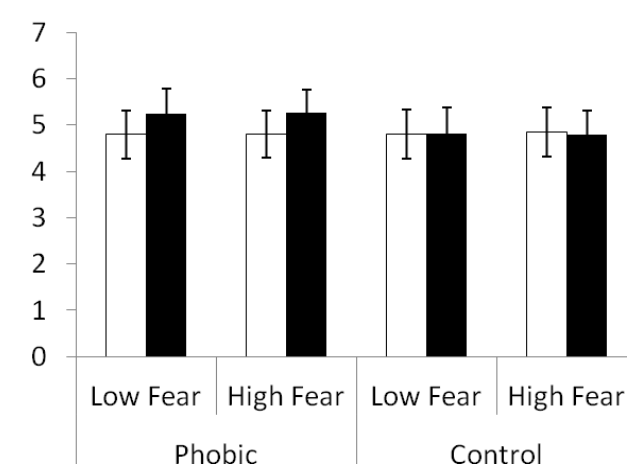

EMG cheek region

in volts (logarithmic transformation)

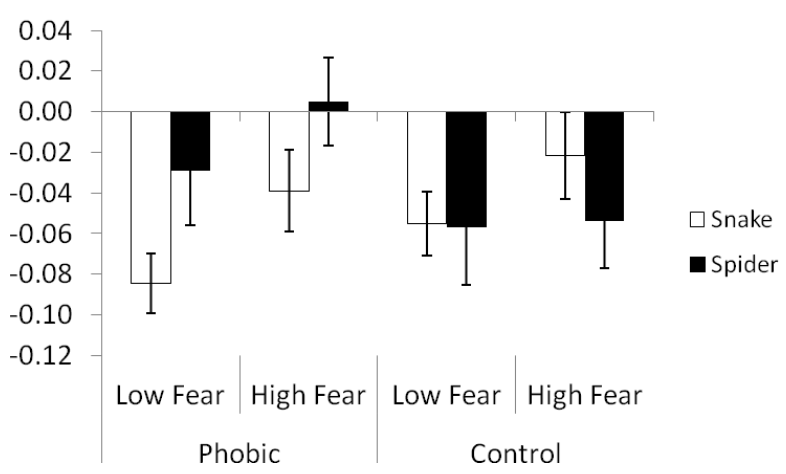


Sensitivity to fear intensity 43

\section{EMG cheek region}

in volts (logarithmic transformation)

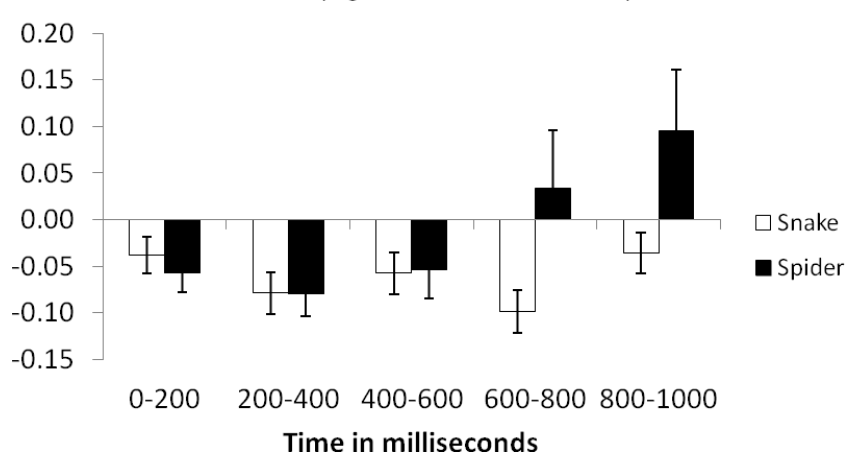

